Case Report

\title{
Unusual Presentation of Rapunzel Syndrome in an 8-Year-Old Girl
}

\author{
Alaa H Rostom MD', Mohammed S Alisi MD², Mai M Akila MD ${ }^{3}$ \\ ${ }^{1}$ Department of General Surgery, An-Najah National University, Nablus, Palestine \\ ${ }^{2}$ Department of Special Surgery, The University of Jordan, Amman, Jordan \\ ${ }^{3}$ Department of Pediatrics, An-Najah National University, Nablus, Palestine
}

Corresponding Author : Mohammed S Alisi MD University of Jordan School of Medicine Queen Rania Al Abdullah St 266 Al Jubaiha, Amman, Jordan 11942

\begin{abstract}
Introduction: Trichobezoar is a rare clinical entity in which a ball of hair accumulates within the alimentary tract. It can be found as an isolated mass in the stomach or may extend into the duodenum and jejunum, which is labeled as Rapunzel syndrome. In this article, we report a rare case of Rapunzel syndrome with a long tail that presented with non-specific features.

Case Presentation: An 8-year-old female patient was referred to our clinic due to palpable abdominal mass. Physical examination revealed non-tender, well-defined abdominal mass and signs of anemia. Imaging showed a giant bezoar filling the stomach, duodenum, and part of jejunum without features of mechanical obstruction. Laparotomy revealed large trichobezoar with long tail. Retrospective evaluation showed significant hair loss and frequent habit of trichotillomania and trichophagia.

Conclusion: Rapunzel Syndrome is not a fairy tale. It may present with vague non-obstructing features. Significant hair loss and underlying psychiatric disorders should raise suspicion to do further investigations. Long-term follow-up and psychiatric management are recommended to prevent recurrence.
\end{abstract}

Keywords: Rapunzel syndrome; abdominal mass; trichobezoar; trichophagia

\section{Introduction}

Bezoars are concretions in the gastrointestinal tract that increase in size by continuous accumulation of non-absorbable food or fibers. It is more common to find this problem in patients who underwent gastric surgery or in those with psychiatric issues. Aggregation of intraluminal nondigestible substances can lead to gastric or small bowel obstruction.

There are several types of bezoar, it can be classified according to the main content or location. Trichobezoar is a rare clinical condition in which a ball of hair accumulates within the gastrointestinal tract. It can either be found as a single mass in the stomach or may extend distally to the duodenum and the jejunum, which is more rare entity labeled as Rapunzel syndrome.

In the absence of altered gastrointestinal anatomy, psychiatric diseases such as trichotillomania and trichophagia may lead to the occurrence of trichobezoars in the stomach.
In this report, we present a case with unusual presentation of Rapunzel syndrome which was rarely reported before in the literature.

\section{Case presentation}

An 8-year-old female child was referred to the pediatric surgery clinic due to palpable abdominal mass. The family denied vomiting or change in bowel habits. Her past medical history includes refractory iron deficiency anemia and failure to gain weight despite treatment by pediatric team. Upon physical examination, she looked pale and malnourished. The abdomen was mildly distended, but soft and non-tender. There was a mobile well-defined lump occupying the epigastric area with right upper quadrant extension. This mass was firm in consistency, not tender, not pulsatile. Normal bowel sounds were present.

Noticeably, she has a considerable loss of hair in the frontal area of the scalp. 
The routine laboratory tests showed anemia (Hemoglobin 7.1g/dl) and thrombocytosis (Platelets count 598 x $10^{9} / \mathrm{L}$ ). No other abnormalities were detected.

The abdomen radiograph was unremarkable. Ultrasonography of the abdomen showed an overdistended stomach with a floating foreign body inside causing displacement of the surrounding structures. There was wall thickening of distal part of stomach and duodenum associated with multiple regional lymph nodes around the stomach.

Abdominal CT scan with IV contrast showed a dilated stomach with large, well-defined, heterogeneous, non-enhancing lesion with contiguous spreads into the duodenum and proximal jejunum (Figure 1).
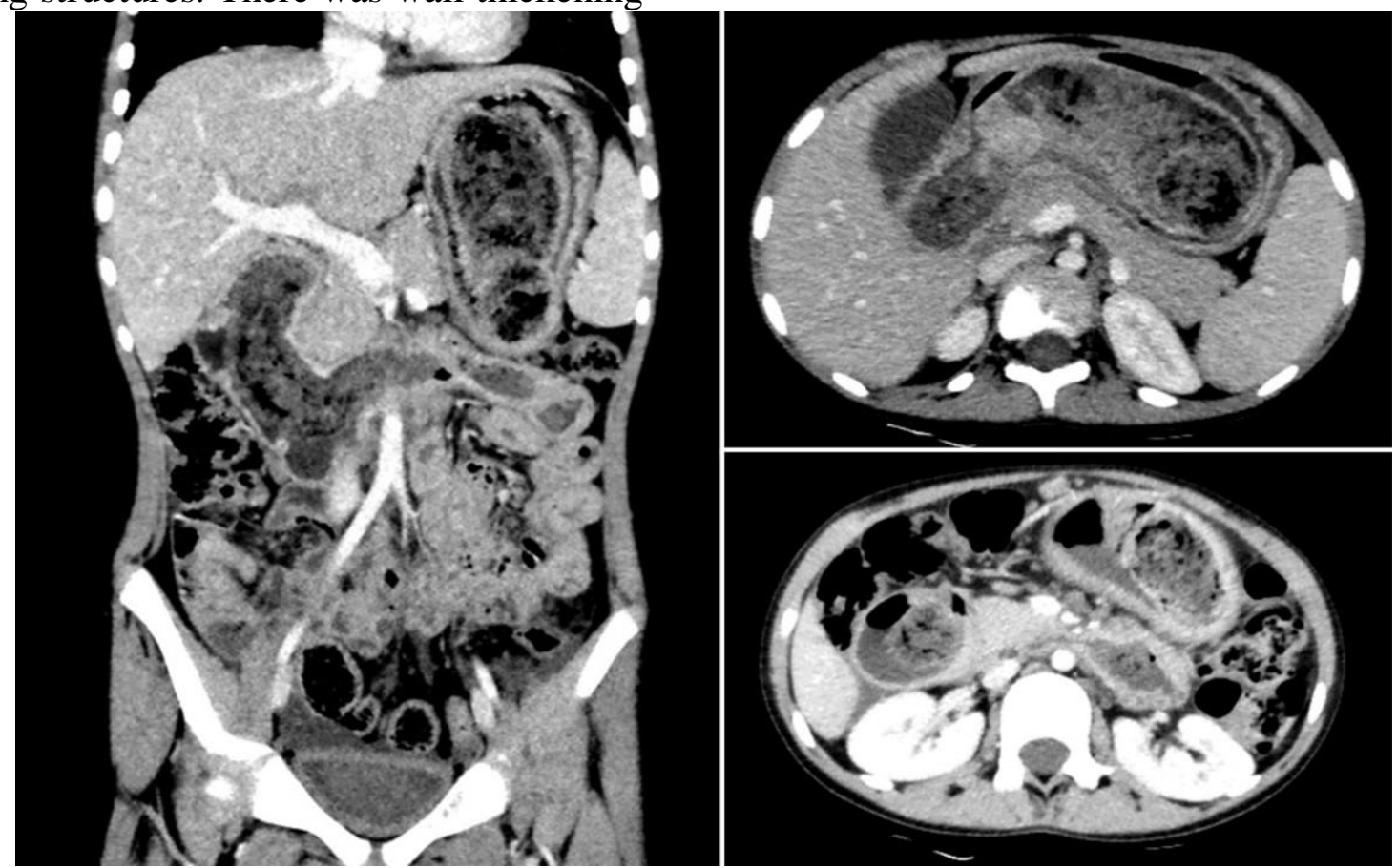

Figure 1: Coronal (left) and axial (right) views abdomen CT scan with IV contrast showed large bezoar in the stomach with extension into the duodenum and jejunum.

The patient underwent surgical exploration via midline laparotomy incision followed by a gastrostomy to find a large mass of hairy bezoar. It was extracted as a single piece through the anterior wall of the stomach (Figure 2). Hence, the diagnosis of Rapunzel syndrome was established.

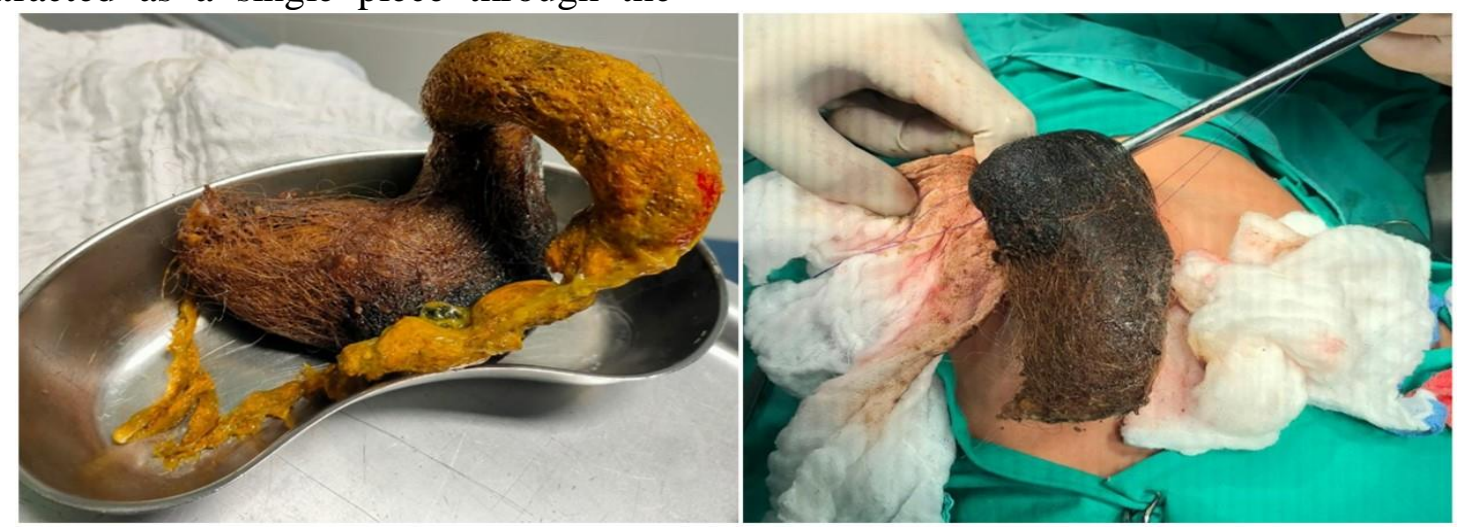

Figure 2: Intraoperative finding of trichobezoar that formulate the shape of stomach and duodenum with jejunal extension

The postoperative course was uneventful and the patient was discharged home after 7 days of hospitalization.
Retrospectively, the family declared the patient's habit of frequent hair-pulling and swallowing in the last one year. 
Psychiatry consultation was started for evaluation and management regarding trichotillomania and trichophagia.

\section{Discussion}

Rapunzel syndrome is a very rare type of trichobezoar. It was first reported in 1968 by Vaughan et al [1]. This was termed so after a fairy tale written in 1812 by Brothers Grimm's about the German princess 'Rapunzel' who let her long golden hair down from her prison tower to permit her young prince to climb up to the window and rescue her [2].

Rapunzel syndrome is commonly found in young adolescent females. It is usually associated with an underlying psychiatric disorder. It forms when a long tail of hair fiber extends from the bulky trichobezoars in the stomach to the small intestine and maybe more distally.

Trichobezoars can present with a vague symptom in the initial phases. In the late stages, the patient may present with symptoms of chronic abdominal pain, gastric ulcer, bleeding, perforation, and picture of intestinal obstruction [3]. Upper gastrointestinal obstruction is the most common manifestation of Rapunzel syndrome. Despite the longstanding history of trichophagia in our case, the presentation was vague. The palpable abdominal mass was the only alarming sign to do further investigations.

Clinical evaluation usually showed a well-defined mobile abdominal mass in about $85 \%$ of cases. Alopecia, hypoalbuminemia, and anemia have also been reported $[4,5]$.

Pathophysiology of trichobezoars formation starts when ingested hair fibers are kept for long time in the mucosa of stomach, gastrointestinal peristalsis makes this fiber to be enmeshed until formation of the ball. The hairy mass progresses in size enough to cause gastric debility. Regardless of the color of the ingested hair, usually, the trichobezoars are black due to the enzymatic oxidation of gastric acid on the hair fibers and often had an unpleasant odor due to fermentation of the fat [6].

In non-complicated cases, the blood investigations are usually within the normal ranges. Some patients may develop iron deficiency anemia and hypoalbuminemia due to malabsorption.

Diagnostic studies include ultrasound, computerized tomography (CT), and upper gastroscopy. Computed axial tomography has the highest accuracy rate.
Ultrasound can exhibit a band of hyperechogenicity formed by the ingested hair fibers mixed with food and air in the trichobezoar. Rate of detection by ultrasound study has been described to be around $88 \%$ [7].

Despite high detection rate of ultrasound study, CT scan remains the study of choice. It can demonstrate a well-defined spherical intraluminal lesion mixed with air bubbles retained within the interstice or a homogenous mottled appearance in the region of the stomach or intestine [8].

Evaluation of our case started with ultrasound followed by CT scan with IV contrast. They showed characteristic finding of Rapunzel syndrome.

Rapunzel syndrome should be considered in the differential diagnoses of young female patients who present with signs of anemia and vague abdominal pain/discomfort. The possibility of diagnosing Rapunzel syndrome increased if the clinician notices signs of hair loss and enquires thoroughly about psychiatric symptoms such as trichotillomania and trichophagia. In 2007, Naik et al [9] reviewed the literature and summarized 24 cases. They concluded that the majority of patients/families deny presence of trichotillomania or trichophagia, even when they certainly asked. The family of our patient declared these symptoms postoperatively.

Successful management of Rapunzel syndrome includes surgical extraction of the trichobezoar and prevention of recurrence through behavioral and psychiatric treatment of the patient.

Non-surgical extraction using enzymatic destruction (pancreatic lipase, cellulose, or papain syrup), extracorporeal shock wave fragmentation, or Nd-YAG laser have been failed to show satisfactory results and usually associated with severe complications [10].

Laparoscopic removal can be applied for small to moderate-sized bezoars, but larger ones $(>20 \mathrm{~cm})$ need removal by more invasive surgical intervention laparotomy.

Endoscopic removal of trichobezoars has been reported. Gorter et al [11] demonstrated that endoscopic extraction of trichobezoars was applicable in only about $5 \%$ of patients, $75 \%$ of laparoscopic removal were successful, and $99 \%$ of all laparotomy followed by gastrotomy cases were successful.

Indeed, laparotomy is a reliable option due to its benefits including short time of procedure, less complication rate, feasibility of exploration of 
other masses or lesions in the alimentary tract, and high success rate. This approach could be the treatment of choice for huge bezoars, though, endoscopic or laparoscopic options can be preferred for smaller ones [12].

Regarding our case, the trichobezoar was large enough to go directly for laparotomy option, a small antral gastrotomy was done via a midline laparotomy incision that enhanced en-bloc removal of the trichobezoar without intraoperative complication.

Recurrence of Rapunzel syndrome has been reported. Long-term clinical follow-up in addition to proper psychiatric consultation is essential to prevent recurrences [13]. In our case, psychiatric evaluation was started before hospital discharge and followed in the clinic.

\section{Conclusion}

Rapunzel Syndrome is not a fairy tale. It is usually affecting young females and may present with non-obstructing or vague features. Significant hair loss and underlying psychiatric disorders should raise suspicion for further investigations. Abdominal CT scan remains the imaging of choice for diagnosis. Extraction via laparotomy is the most successful treatment modality for larger ones. Long-term follow-up and psychiatric management are recommended to prevent recurrence.

Consent: The patient and his/her family signed a written consent to publish their data and images.

Conflicts of interest: None declared.

Role of funding: This research did not receive any specific grant from funding agencies in the public, commercial, or not-for-profit sectors.

\section{References}

[1] Vaughan ED Jr, Sawyers JL, Scott HW Jr. The Rapunzel syndrome. An unusual complication of intestinal bezoar. Surgery. 1968;63(2):339-343.

[2] Kumar N, Huda F, Gupta R, Payal YS, Kumar U, Mallik D. Rapunzel syndrome in adult with mysterious presentation: a rare case report with literature review. Trop Doct. 2019;49(2):133-135. doi: $10.1177 / 0049475519826477$

[3] Williams RS. The fascinating history of bezoars. Med J Aust. 1986;145(1112):613-614.
[4] Hamid M, Chaoui Y, Mountasser M, et al. Giant gastric trichobezoar in a young female with Rapunzel syndrome: case report. Pan Afr Med J. 2017;27:252. Published $2017 \quad$ Aug 4. doi:10.11604/pamj.2017.27.252.9110

[5] Sah DE, Koo J, Price VH. Trichotillomania. Dermatol Ther. 2008;21(1):13-21. doi:10.1111/j.15298019.2008.00165.x

[6] Barzilai M, Peled N, Soudack M, Siplovich L. Harefuah. 1998;135(3-4):97167.

[7] Ripollés T, García-Aguayo J, Martínez MJ, Gil P. Gastrointestinal bezoars: sonographic and CT characteristics. AJR Am J Roentgenol. 2001;177(1):65-69. doi:10.2214/ajr.177.1.1770065

[8] Ersoy YE, Ayan F, Ayan F, Ersan Y. Gastro-intestinal bezoars: thirty-five years experience. Acta Chir Belg. 2009;109(2):198-203. doi:10.1080/00015458.2009.11680405

[9] Naik S, Gupta V, Naik S, et al. Rapunzel syndrome reviewed and redefined. Dig Surg. 2007;24(3):157-161. doi:10.1159/000102098

[10] Nirasawa $Y$, Mori T, Ito $Y$, Tanaka H, Seki N, Atomi Y. Laparoscopic removal of a large gastric trichobezoar. J Pediatr Surg. 1998;33(4):663-665. doi:10.1016/s00223468(98)90342-6

[11] Gorter RR, Kneepkens CM, Mattens EC, Aronson DC, Heij HA. Management of trichobezoar: case report and literature review. Pediatr Surg Int. 2010;26(5):457463. doi:10.1007/s00383-010-2570-0

[12] Fraser JD, Leys CM, St Peter SD. Laparoscopic removal of a gastric trichobezoar in a pediatric patient. $\mathbf{J}$ Laparoendosc Adv Surg Tech A. 2009;19(6):835-837. doi:10.1089/lap.2008.0367

[13] Nettikadan A, Ravi MJ, Shivaprasad M. Recurrent Rapunzel syndrome - A rare tale of a hairy tail. Int $\mathbf{J}$ Surg Case Rep. 2018;45:83-86. doi:10.1016/j.ijscr.2018.03.017 